\title{
Molecular Arrays and Patterns for Supramolecular Materials
}

\author{
Katsuhiko Ariga \\ World Premier International (WPI) Research Center for Materials \\ Nanoarchitectonics (MANA), National Institute for Materials Science (NIMS), 1-1 \\ Namiki, Tsukuba 305-0044, Japan \\ ARIGA.Katsuhiko@nims.go.jp
}

In this article, our recent approaches for preparation of supramolecular materials including molecular array and pattern formation are summarized, as classified by four categories: (i) molecule to nano; (ii) nano to micro; (iii) micro to bulk; (iv) bridging molecule and bulk. Self-assembled processes on a surface provides molecular arrays that can be tuned by several factors such as conformational changes and hydrogen bonding. Microscopic structures can be obtained through self-assembly of molecules designed upon a novel concept of amphiphilicity. Combining self-assembly with layer-by-layer adsorption methods resulted in functional layered micro-structures. By structural transcription from self-assembled structure, bulk materials with precise interior structures such as carbon nanocage can be obtained. The air-water interface provides medium to manipulate functional molecules by bulk visible-scale mechanical motions.

Keywords: Supramolecular materials, self-assembly, structural transcription, molecular recognition.

\section{Introduction}

Nano-structures and micro-structures of functional materials have unavoidable contributions to rapid development of current science and technology. Fabrication technologies, mainly based on so-called top-down approaches, for hard and strong inorganic substances have well developed and provided successful results as seen in silicon-based nanotechnologies. In contrast, soft organic and biological materials including photo-functional polymers and photo-responsible molecules are not always capable to accept these fabrication techniques. Hence, bottom-up approaches based on supramolecular self-assembling processes become important for fabrication of soft nano- and micro-materials $[1,2]$. Obtained materials can be called "supramolecular materials". In this article, our recent approaches for preparation of supramolecular materials including molecular array and pattern formation are summarized, as classified by four categories: (i) molecule to nano; (ii) nano to micro; (iii) micro to bulk; (iv) bridging molecule and bulk.

\section{Molecule to Nano}

Hill and coworkers have investigated multi-color porphyrins useful for versatile anion sensing [3], and recently subjected the porhyrin derivatives to molecular array formation with the aid of Wakayama. Porphyrin regular arrays could be also interesting targets for photo-functions such as light-harvesting systems.

Molecular array of porphyrin molecules, fabricated by vapor deposition onto $\mathrm{Cu}(111)$ surface, were investigated by scanning tunneling microscopy (STM) under ultra-high vacuum. A tetraphenylporphyrin derivative with tert-butyl groups at meta-position and hydroxyl group at para-position of every phenyl ring forms molecular array in hexagonal packing at low temperature, which shifted into square arrangement as temperature elevation (Fig. 1) [4, 5]. Molecular conformation in the hexagonal phase is not most favorable one, but inter-molecular interaction in well-condensed hexagonal phase compensates disadvantages in conformational energies. On the 

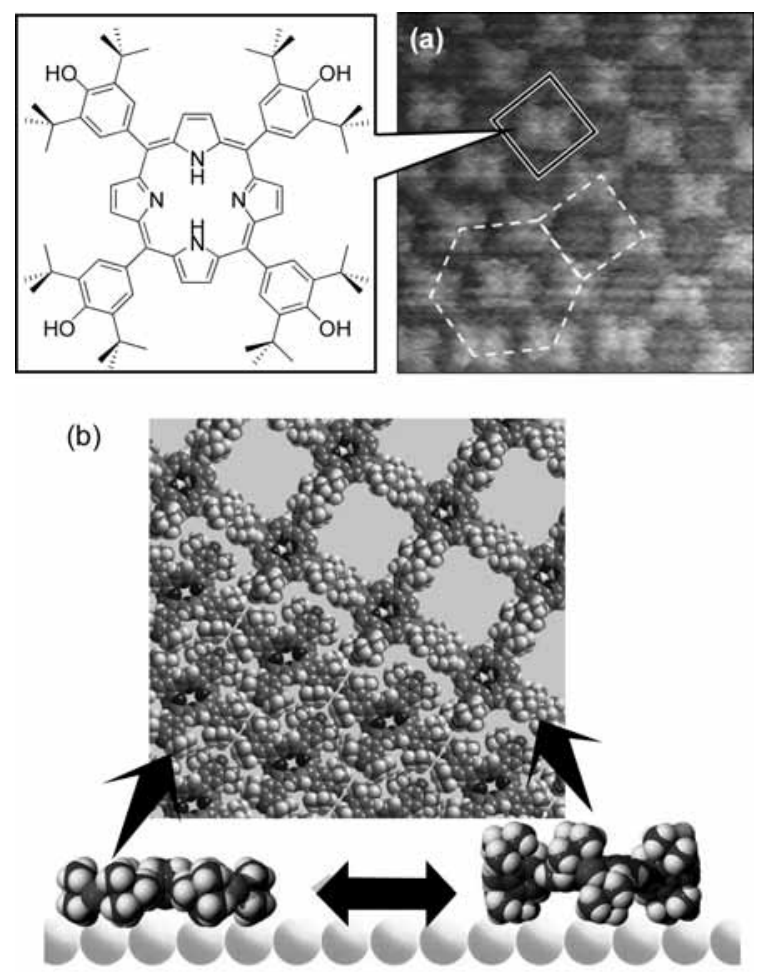

Figure 1 Molecular array of porphyrin derivative with transition from hexagonal to square phase: (a) STM image; (b) molecular model

other hand, the square phase provides most stable conformation for the porphyrin derivative although the molecules are not in well-packed arrangement. Transition between these two phases occurs reversibly in cascade fashion. In separate experiments, coexistence of packed phases consist of flat conformation and rectangle conformation was detected. Surprisingly, boundary of these two phases was filled with a single molecular line conformation-hybrid molecules (half \& half flat/rectangle conformation) [6]. This result suggest that molecules themselves can adjust structural discrepancy between different phases.

In order to introduce expectable molecular interaction to molecular array formation, a new tetraphenylporphyrin derivative with small methyl groups on the phenyl rings was subjected to the molecular array study [7]. Because of less steric hindrance of the methyl substituents, this porphyrin derivative can form hydrogen bond network on a $\mathrm{Cu}$ surface. As the results, formation of Kagomé lattice was clearly confirmed by the
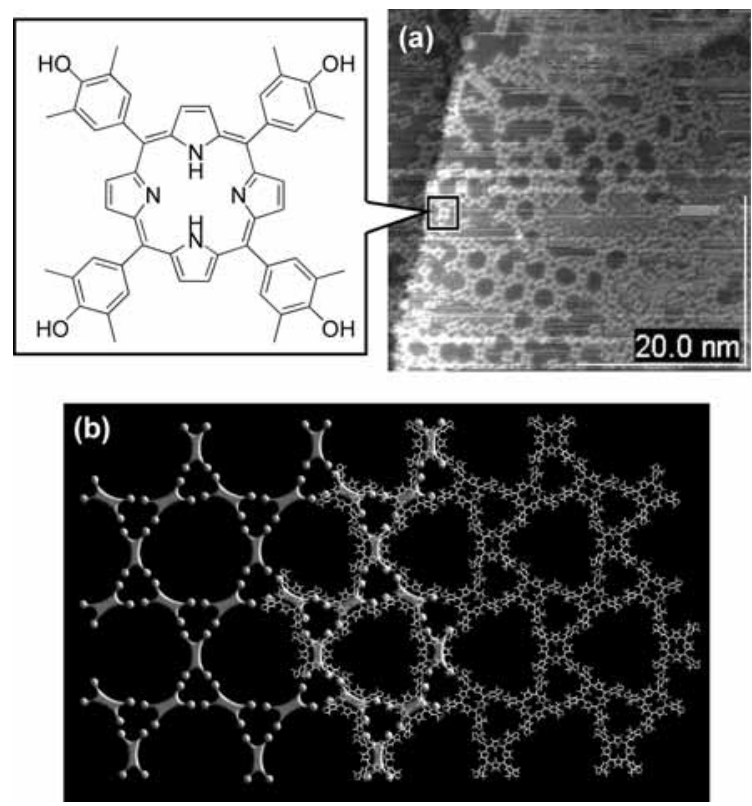

Figure 2 Hydrogen-bonded molecular array (Kagomé lattice) of porphyrin derivative: (a) STM image; (b) molecular model

STM observation (Fig. 2). This result suggests that appropriate design of organic molecules can lead to formation of nano-patterns within two dimensional plane.

Not limited to these examples, we have been proposing various methods to create two-dimensional molecular arrays and patterns. For example, Nakanishi et al reported that a spin-coating of fullerene derivatives on a HOPG plate provides molecular lines with zig-zag fullerene arrays [8]. Periodic patterns can be also obtainable through molecular recognition at a surface of Langmuir monolayers [9-13].

\section{Nano to Micro}

In order to construct desirable micro-structures, control of self-assembling processes is crucial as seen in various examples [14]. Recently, Nakanishi and coworker has proposed a novel type of amphiphilicity based on faint differences between $\mathrm{sp}^{2}$ - and $\mathrm{sp}^{3}$ - carbon natures to provide various micro-objects from a single compound [15], where a conjugate molecule between $\mathrm{sp}^{2}$-type fullerene and $\mathrm{sp}^{3}$-type alkyl chains was demonstrated to form microscopic tapes, spheres, disks, and cones. Similarly, 


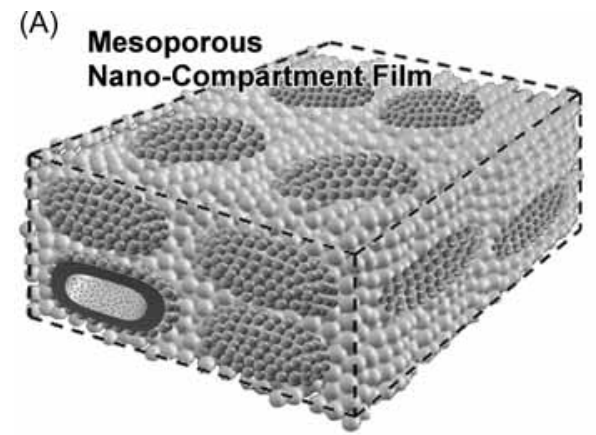

(B)

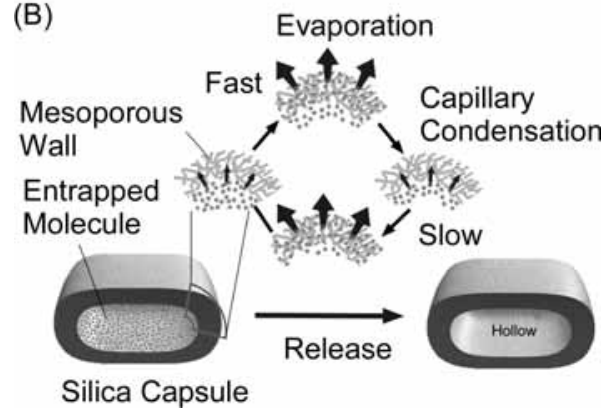

Figure 3 (A) schematic illustration of mesoporous nanocompartment film and (B) step-wise material release mechanism.

flower-like objects in micrometer scale was prepared [16] and subjected to form superhydrophobic surface [17]. It was also demonstrated that oily fullerene materials can be obtained through faint structural modification of the alkyl-fullerene derivatives [18].

For formation of micro-structures with more intentional design, appropriate fabrication techniques have to be combined with self-assembling processes. For example, layer-by-layer (LbL) assembly [19-23], which is regarded as a versatile method for intentional fabrication of the layered structures, is one of the most powerful methods for this purpose. Ji et al. reported a novel type of micro-structured films, so-called "mesoporous nano-compartment films", which are useful for controlled materials release (Fig. 3) [24]. The resulting mesoporous nano-compartment films possess molecular encapsulation and release capabilities. With these films, stimuli-free auto-modulated stepwise release of water or drug molecules was achieved through the mesopore channels of robust silica capsule containers embedded in the film. The stepwise release of water was reproducibly observed, which may be originated from the non-equilibrated rates between evaporation of water from the mesopore channels to the exterior and the capillary penetration of water from container interior to the mesopore channels. This behavior was generalized to evaporation of other substance, fragrances, limonene. The controlled release of the sunscreen UV-absorber (UV-S1) for circumvention of its rapid dissolution in water and prolongation of its prophylactic effect towards harmful ultraviolet radiation were also investigated. These mesoporous nano-compartment films will be promising materials for drug delivery since they allow gradual release of therapeutic agents with likely related improvements in their efficacy.

\section{Micro to Bulk}

Fabrication of bulk materials reflecting regular nano- and micro-structures is also attractive targets in the field of supramolecular materials. methods so-called template synthesis and/or structure transcription are powerful methods for this target. For example, preparation of mesoporous materials using templates of molecular assemblies and nanostructured materials could be one of the most successful examples [25-35].

Vinu and coworkers recently applied this concept to prepare novel mesoporous materials [36], including mesoporous carbon nitride [37], mesoporous boron nitride, and mesoporous boron carbon nitride [38]. Especially, novel supramolecular material, carbon nanocage [39-41], showed interesting functions (Fig. 4). Carbon nanocage was synthesized using cage-type mesoporous silica (KIT-5) as a hard template through controlled pore-filling technique and has regular mesopores (see TEM image in Fig. 4A) with pore diameter of $5.2 \mathrm{~nm}$ and cage diameter of $15.0 \mathrm{~nm}$. This material has huge surface area (1600 $\left.\mathrm{m}^{2} \mathrm{~g}^{-1}\right)$ and pore volume $\left(2.1 \mathrm{~cm}^{3} \mathrm{~g}^{-1}\right)$, as compared with conventional mesoporous carbon, CMK-3. As shown in Fig. 4B, the carbon nanocage material exhibited excellent adsorption capacities for a typical dye (Alizarin Yellow), where only carbon nanocage is capable of perfect removal of the dye stuff [42]. Competitive adsorption of catechin and tannic acid to the carbon nanocage material resulted in highly selective adsorption of tannic acid (ca. 95\%) using a very simple one-pot process. This could be simple, economical, and 

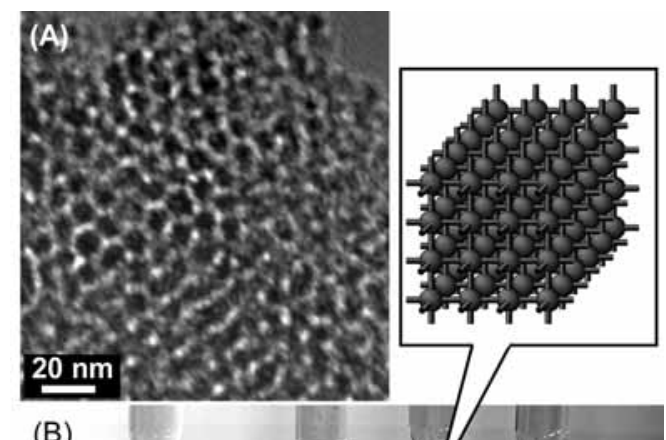

(B)

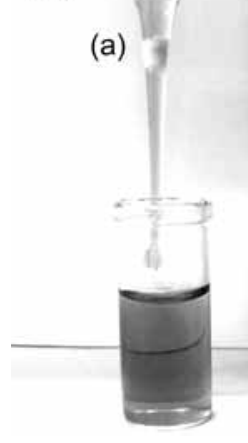

(b)

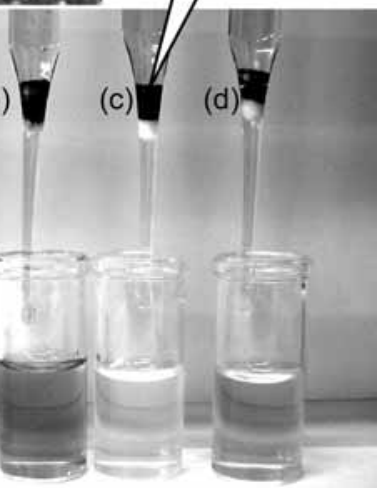

Figure 4 (A) TEM image of carbon nanocage. (B) Dye filtration experiment: (a) no carbon; (b) activated carbon; (c) carbon nanocage; mesoporous carbon CMK-3

toxic-solvent-free one-pot separation of various materials.

\section{Bridging Molecule and Bulk}

A novel methodology to bridge molecular function and bulk motion has been also developed through molecular recognition at the air-water interface. The air-water interface is regarded as medium appropriate for molecular recognition of various aqueous guests [43-51] and allows dynamic control of conformational changes and motions of embedded molecules. Using these characteristics, we successfully manipulated molecules at the air-water interface upon bulk (10-100 cm size) motion of the entire monolayer and realized "capture and release" of aqueous guest molecules [52-56].

As shown in Fig. 5A, the system utilizes monolayers of a steroid cyclophane, which contains

1,6,20,25-tetraaza[6.1.6.1]-paracyclophane cyclic core connected to four steroid moieties (cholic acid) through a flexible L-lysine spacer.
(A)

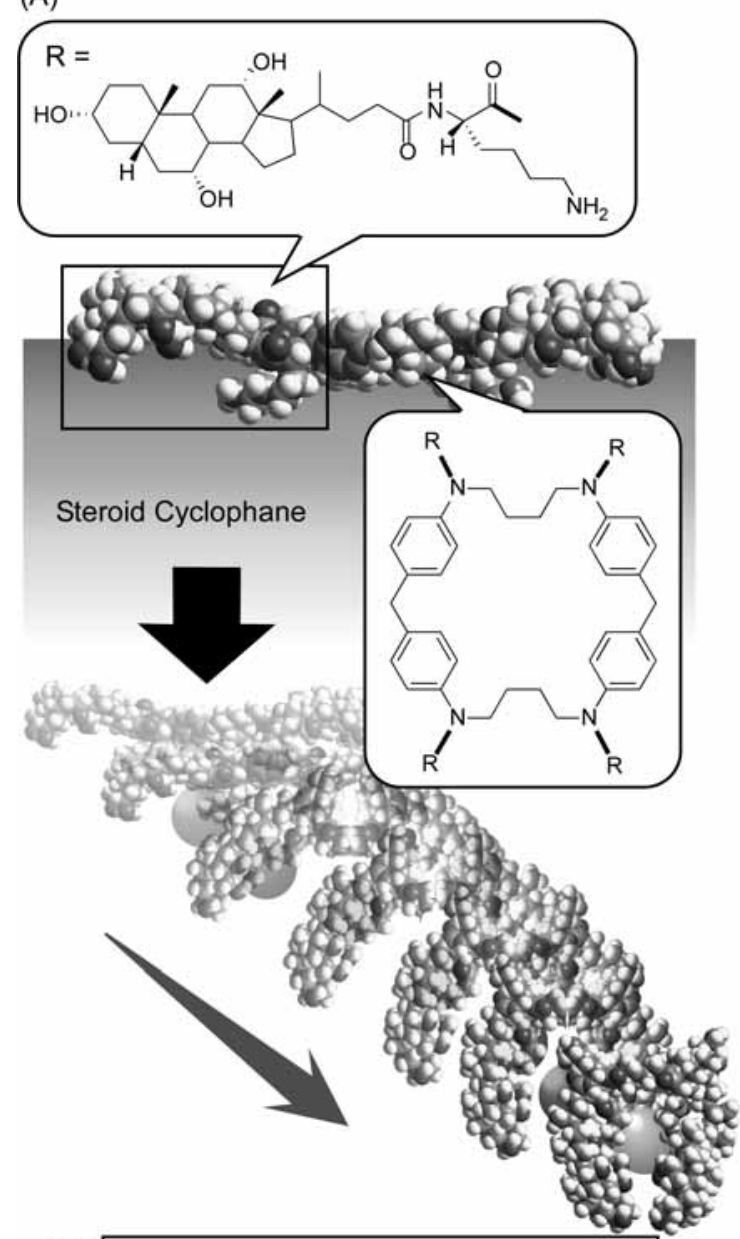

(B)

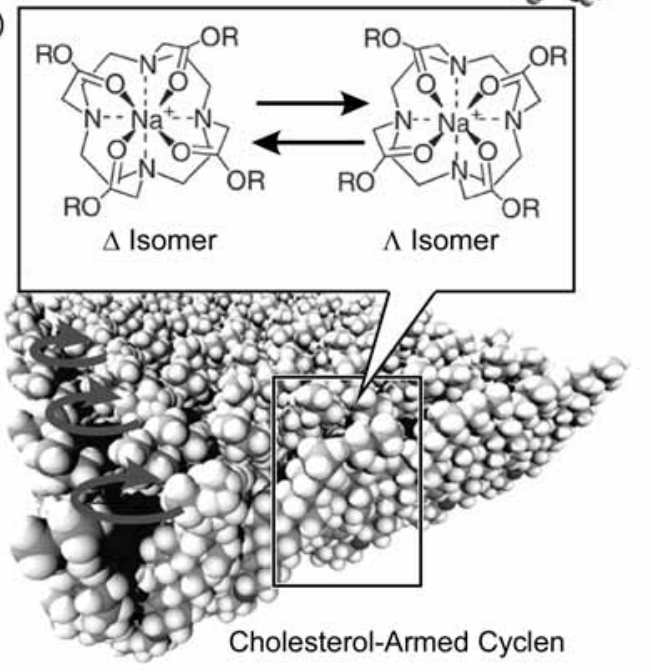

Figure 5 Dynamic molecular recognition by (A) steroid cyclophane and (B) cholesterol-armed cyclen. 
Compression and expansion of the monolayer of steroid cyclophane induces reversible formation of the cavity conformation resulting in reversible binding (capture and release) of an aqueous fluorescent guest by a repeated compression and expansion of the steroid cyclophane monolayer.

Another example is based on molecular twisting as shown in Fig. 5B, where an octacoordinate sodium complex of a polycholesteryl-substituted cyclen embedded at the air-water interface was used as a molecular host [57]. This complex has two possible quadruple helicate structures and its helicity is influenced by the chirality of the side arms especially when ordered or aggregated at the supramolecular level. Therefore, chiral selectivity on guest binding can be modulated by monolayer compression. The binding constant of $\mathrm{L}$-valine is smaller than that of D-valine at low surface pressure but exceed them at $22-23 \mathrm{mN} \mathrm{m}^{-1}$. This indicates that chiral recognition of valine stereoisomers by the cyclen monolayer reverses from the $\mathrm{D}$ - to $\mathrm{L}$-form upon monolayer compression.

\section{Summary}

Fabrication and function of supramolecular materials that have been developed in our research team are briefly summarized above. Key concepts in construction of supramolecular materials are self-assembly and structural transcription. In addition, hierarchic design and dynamic controls of supramolecular materials would provide novel functional development. The described approaches can be, of course, applicable to photopolymers to create novel photonic functions.

\section{Acknowledgment}

The researches described in this article were partially supported by Grant-in-Aid for Scientific Research on Priority Areas "Chemistry of Coordination Space" and a Grant-in-Aid for Science Research in a Priority Area "Super-Hierarchical Structures" from Ministry of Education, Science, Sports, and Culture, Japan, and Grants-in-Aid for Scientific Research (B) from Japan Society for the Promotion of Science.

\section{References}

1. K. Ariga, T. Nakanishi and T. Michinobu, $J$. Nanosci. Nanotechnol. 6 (2006) 2278.

2. Y. Xie, J. P. Hill, R. Charvet and K. Ariga, $J$. Nanosci. Nanotechnol. 7 (2007) 2969.

3. J. P. Hill, A. L. McCarty, F. D'Souza, J. Labuta, C. Redshaw, M. R. J. Elsegood, M. Aoyagi, T. Nakanishi and K. Ariga, Inorg. Chem. 45 (2006) 8288.

4. J. P. Hill, Y. Wakayama, W. Schmitt, T. Tsuruoka, T. Nakanishi, M. L. Zandler, A. L. McCarty, F. D'Souza, L. R. Milgrom and K. Ariga, Chem. Commun. (2006) 2320.

5. Y. Wakayama, J. P. Hill and K. Ariga, Surf. Sci. 601 (2007) 3984.

6. J. P. Hill, Y. Wakayama and K. Ariga, Phys. Chem. Chem. Phys. 8 (2006) 5034.

7. J. P. Hill, Y. Wakayama, M. Akada and K. Ariga, J. Phys. Chem. C 111 (2007) 16174.

8. T. Nakanishi, N. Miyashita, T. Michinobu, Y. Wakayama, T. Tsuruoka, K. Ariga and D. G. Kurth, J. Am. Chem. Soc. 128 (2006) 6328.

9. H. Koyano, K. Yoshihara, K. Ariga, T. Kunitake, Y. Oishi, O. Kawano, M. Kuramori and K. Suehiro, Chem. Commun. (1996) 1769.

10. Y. Oishi, Y. Torii, T. Kato, M. Kuramori, K. Suehiro, K. Ariga, K. Taguchi, A. Kamino, H. Koyano and T. Kunitake, Langmuir 13 (1997) 519.

11. Y. Oishi, T. Kato, M. Kuramori, K. Suehiro, K. Ariga, A. Kamino, H. Koyano and T. Kunitake, Chem. Commun. (1997) 1357.

12. K. Ariga, J. Nanosci. Nanotechnol. 4 (2004) 23.

13. Y. Oishi, T. Kato, T. Narita, K. Ariga and T. Kunitake, Langmuir 24 (2008) 1682.

14. K. Ariga, T. Nakanishi and J. P. Hill, Curr. Opin. Colloid Interface Sci. 12 (2007) 106.

15. T. Nakanishi, W. Schmitt, T. Michinobu, D. G. Kurth and K. Ariga, Chem. Commun. (2005) 5982.

16. T. Nakanishi, K. Ariga, T. Michinobu, K. Yoshida, H. Takahashi, T. Teranishi, H. Möhwald and D. G. Kurth, Small 3 (2007) 2019.

17. T. Nakanishi, T. Michinobu, K. Yoshida, N. Shirahata, K. Ariga, H. Möhwald and D. G. Kurth, Adv. Mater. 20 (2008) 443. 
18. T. Michinobu, T. Nakanishi, J. P. Hill, M. Funabashi and K. Ariga, J. Am. Chem. Soc. 128 (2006) 10384.

19. Y. Lvov, K. Ariga, I. Ichinose and T. Kunitake, J. Am. Chem. Soc. 117 (1995) 6117.

20.K. Ariga, Y. Lvov, I. Ichinose and T. Kunitake, App. Clay Sci. 15 (1999) 137.

21. K. Katagiri, R. Hamasaki, K. Ariga and J. Kikuchi, Langmuir 18 (2002) 6709.

22. K. Katagiri, R. Hamasaki, K. Ariga and J. Kikuchi, J. Am. Chem. Soc. 124 (2002) 7892.

23. K. Ariga, J. P. Hill, and Q. Ji, Phys. Chem. Chem. Phys. 9 (2007) 2319.

24. Q. Ji, M. Miyahara, J. P. Hill, S. Acharya, A. Vinu, S. B. Yoon, J.-S. Yu, K. Sakamoto and K. Ariga, J. Am. Chem. Soc. 130 (2008) 2376.

25. A. Okabe, T. Fukushima, K. Ariga, and T. Aida, Angew. Chem., Int. Ed. 41 (2002) 3414.

26. Q. Zhang, K. Ariga, A. Okabe and T. Aida, $J$. Am. Chem. Soc. 126 (2004) 988.

27. K. Ariga, Chem. Rec. 3 (2004) 297.

28. A. Okabe, T. Fukushima, K. Ariga, M. Niki and T. Aida, J. Am. Chem. Soc. 126 (2004) 9013.

29. A. Vinu, K. Z. Hossain and K. Ariga, $J$. Nanosci. Nanotechnol. 5 (2005) 347.

30. A. Vinu, M. Miyahara and K. Ariga, J. Phys. Chem. B 109 (2005) 6436.

31. H. Jin, Z. Liu, T. Ohsuna, O. Terasaki, Y. Inoue, K. Sakamoto, T. Nakanishi, K. Ariga and S. Che, Adv. Mater. 18 (2006) 593.

32. M. Miyahara, A. Vinu and K. Ariga, $J$. Nanosci. Nanotechnol. 6 (2006) 1765.

33. W. Otani, K. Kinbara, Q. Zhang, K. Ariga and T. Aida, Chem. Eur. J. 13 (2007) 1731.

34. K. Ariga, A. Vinu, J. P. Hill and T. Mori, Coord. Chem. Rev. 251 (2007) 2562.

35. A. Vinu, K. Z. Hossian, P. Srinivasu, M. Miyahara, S. Anandan, N. Gokulakrishnan, T. Mori, K. Ariga and V. V. Balasubramanian, $J$. Mater. Chem. 17 (2007) 1819.

36. A. Vinu, T. Mori and K. Ariga, Sci. Technol. Adv. Mater. 7 (2006) 753.

37. A. Vinu, K. Ariga, T. Mori, T. Nakanishi, S. Hishita, D. Golberg and Y. Bando, Adv. Mater. 17 (2005) 1648.

38. A. Vinu, M. Terrones, D. Golberg, S. Hishita, K. Ariga and T. Mori, Chem. Mater. 17 (2005) 5887.
39. A. Vinu, M. Miyahara, V. Sivamurugan, T. Mori and K. Ariga, J. Mater. Chem. 15 (2005) 5122.

40. A. Vinu, M. Miyahara, T. Mori and K. Ariga, $J$. Porous Mater. 13 (2006) 379.

41. P. Srinivasu, V. V. Balasubramanian, L. Kumaresan, D. P. Sawant, X. Jin, S. Alam, K. Ariga, T. Mori and A. Vinu, J. Nanosci. Nanotechnol. 7 (2007) 3250.

42. K. Ariga, A. Vinu, M. Miyahara, J. P. Hill and T. Mori, J. Am. Chem. Soc. 129 (2007) 11022.

43. K. Ariga, T. Kunitake and H. Furuta, J. Chem. Soc., Perkin Trans. 2 (1996) 667.

44. K. Ariga, A. Kamino, H. Koyano and T. Kunitake, J. Mater. Chem. 7 (1997) 1155.

45. M. Sakurai, H. Tamagawa, Y. Inoue, K. Ariga and T. Kunitake, J. Phys. Chem. B 101 (1997) 4810 .

46. H. Tamagawa, M. Sakurai, Y. Inoue, K. Ariga and T. Kunitake, J. Phys, Chem. B 101 (1997) 4817.

47. K. Ariga and T. Kunitake, Acc. Chem. Res. 31 (1998) 371.

48. V. Marchi-Artzner, F. Artzner, O. Karthaus, M. Shimomura, K. Ariga, T. Kunitake and J.-M. Lehn, Langmuir 14 (1998) 5164.

49. K. Ariga, A. Kamino, X. Cha and T. Kunitake, Langmuir 15 (1999) 3875.

50. K. Ariga, T. Nakanishi, J. P. Hill, M. Shirai, M. Okuno, T. Abe and J. Kikuchi, J. Am. Chem. Soc. 127 (2005) 12074.

51. K. Ariga, T. Michinobu, T. Nakanishi and J. P. Hill, Curr. Opin. Colloid Interface Sci. 13 (2008) 23.

52. K. Ariga, Y. Terasaka, D. Sakai, H. Tsuji and J. Kikuchi, J. Am. Chem. Soc. 122 (2000) 7835.

53. K. Ariga, T. Nakanishi, Y. Terasaka, H. Tsuji, D. Sakai and J. Kukuchi, Langmuir 21 (2005) 976.

54. K. Ariga, T. Nakanishi, J. P. Hill, Y. Terasaka, D. Sakai and J. Kikuchi, Soft Matter 1 (2005) 132.

55. K. Ariga, T. Nakanishi and J. P. Hill, Soft Matter 2 (2006) 465.

56. K. Ariga, T. Nakanishi, Y. Terasaka and J. Kikuchi, J. Porous Mater. 13 (2006) 427.

57. T. Michinobu, S. Shinoda, T. Nakanishi, J. P. Hill, K. Fujii, T. N. Player, H. Tsukube and K. Ariga, J. Am. Chem. Soc. 128 (2006) 14478. 